\title{
CURRENT SCENARIO OF VACCINES FOR JAPANESE ENCEPHALITIS
}

\author{
Shirisha Cherukommu, ML Arvinda Swamy and Shailendra K. Saxena \\ CSIR-Centre for Cellular and Molecular Biology (CCMB), Uppal Road, Hyderabad 500007, India
}

\begin{abstract}
Currently various countries in South-East Asia, including India, are facing disaster due to Acute Encephalitis Syndrome (AES), which is primarily caused by Japanese Encephalitis Virus (JEV). A dirty environment and a lack of awareness about the risks associated with mosquito bites may be one of the reasons of the outbreak. Japanese encephalitis is a lethal neuroviral infection for which there is no treatment and it largely affects children less than 14 years. Most of the cases either remain asymptomatic or shortlived mild symptoms with fever. In less than one case in every 300-500, the virus causes serious symptoms, such as seizures, confusion and paralysis. Up to one in every three people who develop serious symptoms die and those who do survive may suffer permanent neurological damage. However, the disease can be prevented by JE-specific vaccination. The current article tries to create awareness and is a compilation of advances made so far for vaccines for Japanese encephalitis.
\end{abstract}

Keywords: Vaccines, Japanese Encephalitis, Flavivirus

\section{INTRODUCTION}

Japanese encephalitis is the most important epidemic encephalitis in the world, responsible for a higher burden of disability than any other arthropodborne virus. Japanese Encephalitis Virus (JEV) is the member of the family flaviviridae, targets central nervous system, leading to high mortality and neurological and psychiatric sequelae. JEV is prevalent in much of Asia and the Western Pacific, with over 4 billion people living at risk of infection. In the absence of antiviral intervention, vaccination is the only strategy to develop long-term sustainable protection against JEV infection (Yun and Lee, 2014). It is an important viral neurological infection. Although only a minority of people exposed to the virus develop disease, Japanese encephalitis is estimated to be responsible for nearly 70 000 cases and 20000 deaths annually. Patients develop a febrile illness followed by headache, vomiting, impaired consciousness and often seizures. JEV represents the JE serogroup, which also includes West Nile, Murray Valley encephalitis and St. Louis encephalitis viruses.
JEV is a vaccine-preventable pathogen, but the molecular basis of its neurovirulence remains unknown.

\section{VACCINES}

Japanese Encephalitis (JE) virus requires dissemination within the host via the circulation for disease development. Antibodies required for neutralizing the serum would be effective for fighting the disease (Konishi, 2013). The most effective method to control this viral infection currently is by vaccination. Current licensed JE vaccines induce neutralizing antibodies and titers of $1: 10$ or higher are the recommended immunological correlate of protection. There are now two prequalified World Health Organization (WHO) vaccines of which at least one among them can be used by low income countries and a Global Alliance for Vaccines and Immunization 9 GAVI) Alliance commitment to provide financial support to eligible countries for campaigns for children aged 9 months through 14 years (Hills et al., 2014).

Corresponding Author: Shailendra K. Saxena, CSIR-Centre for Cellular and Molecular Biology (CCMB), Uppal Road, Hyderabad 500007, India 


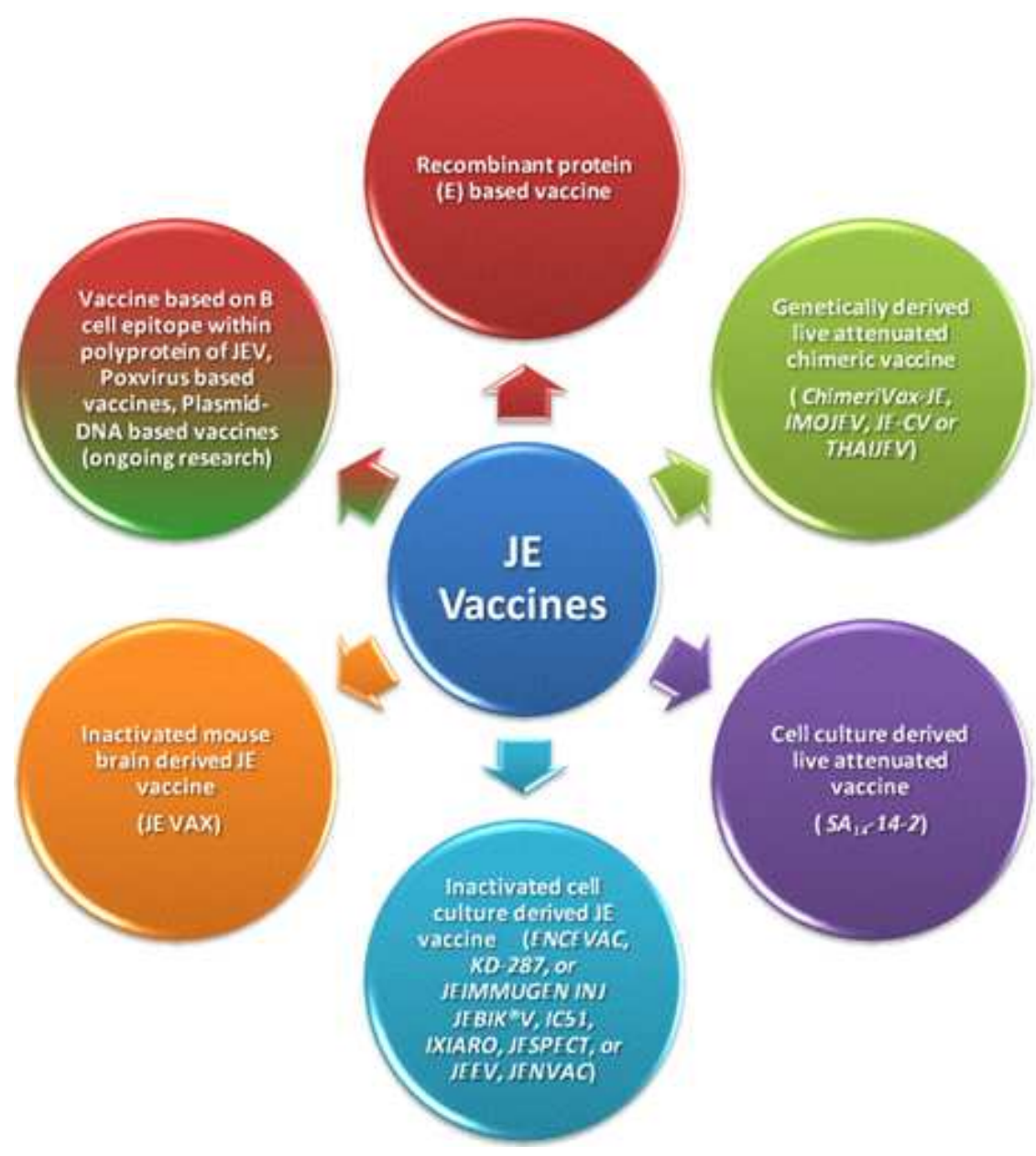

Fig. 1. Types of Japanese encephalitis vaccines available

Because most flavivirus life cycles are maintained between arthropod vectors and amplifying/reservoir hosts in the absence of humans, eradication of flaviviruses might be extremely difficult (Ishikawa et al., 2014). Effective vaccines are also available for the immunoprophylaxis of Japanese encephalitis, including both inactivated whole virus and live attenuated vaccines (Fig. 1). There are many kinds of available vaccines including inactivated mouse brain derived vaccine, inactivated cell culture derived vaccine, cell culture derived live attenuated JE vaccine, genetically engineered live attenuated vaccine and recombinant vaccine. Studies are also going on to find out the B cell epitope within the polyprotein of JEV and target on vaccine preparation that promote B cell activity. Efforts also have been put on developing recombinant and chimeric vaccines. Originally, the substrates for inactivated vaccines were either infected mouse brains or primary hamster kidney cells (China) and vaccine efficacies of 76-95\% were reported.

\subsection{Inactivated Mouse Brain Derived Japanese Encephalitis Vaccine (MB JE Vaccine)}

First vaccine was developed by Japan based on Nakayama strain of JEV. They collected the infected mouse brain homogenate and inactivated with formalin. This strain was used for vaccine development in several Asian countries including Korea, Vietnam and India. Latter, another strain was used named as Beijing-1 which was found to show more efficacy and higher yield during vaccine production (Yun and Lee, 2014). But, studies have shown that there was no much difference in the 
efficacy between these two vaccines. Both Inactivated mouse brain derived JE vaccine and inactivated cell culture derived JE vaccine contains the same Japanese encephalitis virus strain, the Beijing-1 strain. Mouse brain-derived, purified inactivated JE vaccine (MB JE vaccine) has been locally produced and introduced into Thai National Immunization Program (NIP) since 1990. Mouse brain derived inactivated Nakayama vaccine was marketed as JE VAX for several decades (Yun and Lee, 2014). But, this vaccine had drawbacks including vaccine induced adverse events, requires booster doses and high production costs. Over the past half-century, a mouse brain-derived inactivated vaccine has been used internationally for active immunization. The brain material used in vaccine development should be reduced to avoid any neurological complications (Halstead and Thomas, 2010). In recent years, production of the mouse brain-derived vaccine has been discontinued, but 3 new cell culture-derived vaccines are available in various parts of the world.

\subsection{Inactivated Cell Culture Derived Japanese Encephalitis Vaccine (CC-JE Vaccine)}

During the Beijing-1 strain epidemic, Chinese developed a PHK cell derived inactivated vaccine based on Beijing-3(P3) strain (Yun and Lee, 2014). This vaccine was adapted to production in African green monkey kidney (Vero) cells. Vero cellderived inactivated vaccine against Japanese encephalitis was introduced in 2009 by Japan based on Beijing-1 strain with the name JEBIK V and another one ENCEVAC in 2011 (Yun and Lee, 2014). This inactivated cell culture derived JE vaccine when compared to inactivated mouse brain derived JE vaccine, is highly immunogenic showing significantly high geometric mean titres (Jelinek, 2013). Studies on children aged 2 months to 18 years have shown excellent safety profile about this vaccine. To improve the stability, CC-JE Vaccine was converted from a liquid type to a freeze-dried type of vaccine. Freeze dried, cell culture-derived Japanese encephalitis vaccine (Inactivated) (JEBIK®V) is approved for a three-dose primary immunization followed by a one-dose booster immunization in Japan (Okada et al., 2012). For the past several decades, killed viral vaccines prepared in tissue culture or mouse brain have been used effectively to immunize travelers and residents of enzootic countries. In China, the Vero cell-derived Beijing-3 vaccine was licensed in 1998 and is now the leading inactivated vaccine for domestic use, but it is being replaced by the live-attenuated SA14-14-2 vaccine (Yun and Lee, 2014). JENVAC ${ }^{\circledR}$ is an inactivated Vero cellderived vaccine prepared from thermostable Kolar Strain (JEV $821564 \mathrm{XY})$. Data of 2-dose study shows that a single dose of JENVAC is sufficient to elicit the immune response as the subjects who received a single dose were $98.67 \%$ seroprotected and the 4-fold seroconversion was at $93.14 \%$ for the $\leq 50$ to $\leq 1$ year age group.

\subsection{Cell Culture Derived Live Attenuated Vaccine}

Limited access in Asia and safety concerns with regard to mouse brain products prompted the Chinese to develop a live, attenuated virus vaccine $\left(\mathrm{SA}_{14}-14-2\right.$; Chengdu Institute of Biological Products), which has proven to be safe and efficacious following administration of $>300$ million doses (Halstead and Thomas, 2010). China has developed a live-attenuated vaccine that has proven to induce protective immunity following a single inoculation. The cell culture derived live $\mathrm{SA}_{14}$-14-2 Vaccine is based on a stable neuroattenuated strain of JEV $\left(\mathrm{SA}_{14}-14-2\right)$ (Verma, 2012). Cost, efficacy and safety concerns led to the development of a live-attenuated virus vaccine $\left(\mathrm{SA}_{14}-14-\right.$ 2) (Halstead and Thomas, 2011). Here, scientists constructed an infectious cDNA of the most widely used live-attenuated JE vaccine, $\mathrm{SA}_{14}-14-2$ and rescued from the cDNA a molecularly cloned virus, $\mathrm{SA}_{14}-14-2 \mathrm{MCV}$, which displayed in vitro growth properties and in vivo attenuation phenotypes identical to those of its parent, $\mathrm{SA}_{14}-14-2$. $\mathrm{SA}_{14}-14-2$ is highly immunogenic, as shown by the high percentage of seroconversion with 1 dose (85\%-100\%) andnear-complete seroconversion with 2 doses given 1 to 3 months apart (Yun and Lee, 2014). Using this cDNA technology, a single Gly-to-Glu change at E-244 is sufficient to confer lethal neurovirulence in mice, including rapid development of viral spread and tissue inflammation in the central nervous system was shown. This comprehensive site directed mutagenesis showed altered JEV infectivity in vitro as well as with the level of neurovirulence in vivo, in case of both mice and human neuronal cells. But, it had no significant effect on viral RNA replication (Yun et al., 2014). Still, there is a theoretical risk for reversionof the attenuated $\mathrm{SA}_{14}$-14-2 virus to high virulence, which has restricted its extended application to global immunization for the prevention of JEV infection (Yun and Lee, 2014). 


\subsection{Genetically Derived Live Attenuated Chimeric Vaccine}

A recombinant, live-attenuated $\mathrm{JE}$ vaccine based on a chimeric YF-JE virus (designated ChimeriVax-JE) has also been produced. The ChimeriVax-JE is generated by using infectious YFV 17D cDNA technology, in which the genes encoding the prM and E proteins of YFV 17D are replaced with the corresponding genes of JEV SA1414-2 (Yun and Lee, 2014). ChimeriVax-JE virus has been shown to be restricted in its ability to infect and replicate in six different mosquito species following oral feeding of artificial blood meals with high titers of the virus, although all the mosquitoes are susceptible to JEV infection. ChimeriVax-JE virus is thus less likely to be transmitted by mosquitoes from vaccinated persons to other hosts.

\subsection{Recombinant Protein Based Vaccine}

A mouse brain-derived, formalin-inactivated vaccine is available for immunization against JE. Because the formalin-inactivated JEV vaccine and inactivated cell culture derived JE vaccine has limitations in terms of safety, availability and cost, attempts are being made to develop improved vaccine using the recombinant DNA technology (Kaur and Vatic, 2003). A 27 amino acid peptide from envelope protein (E) of JEV is fused with John grass mosaic virus protein to form virus like particles has been developed to produce neutralizing antibodies against JEV infection (Yun and Lee, 2014). Various attempts are made for the development of recombinant yellow fever virus or the plasmid DNA-based JEV vaccine. In addition Chimerivax-JE vaccine, a novel live attenuated one dose chimeric vaccine comprising the structural genes of SA14-14-2virus and nonstructural genes of yellow fever 17D virus, is in the process of getting licensed in Australia and in South-East Asia (Wilder-Smith and Halstead, 2010).

\section{EXPERIMENTAL VACCINES}

Several other vaccines like plasmid-DNA based vaccines (plasmid encoding prM and/or E protein) and poxvirus based vaccines (in which recombinant poxviruses are used as viral vectors in delivering the JEV antigens) are at experimental stages. An important focus proposed by the vaccine experts is to find one vaccine that can promote $\mathrm{B}$ cell activity. The technique to be used is ABCpred Prediction7 (Yasri and Wiwanitkit, 2014). This area mainly targets the immunogenic epitope within the polyprotein of Japanese encephalitis virus. Advanced bioinformatics technique is used to find out the epitopic region within polyprotein of JEV.

\section{ALLERGIC REACTIONS TO JE VACCINE}

In 1900s, several allergic reactions were observed in the people of several countries vaccinated with inactivated mouse brain derived vaccine. Allergic reactions, including the mucocutaneous and neurologic reactions reported after $\mathrm{JE}$ vaccination, may vary in frequency and these reactions should be evaluated meticulously yearly (Plesner, 2003). This shows that vaccine to be developed should have fewer side effects and elicit immune response in a single dose.

\section{CONCLUSION}

Vaccines play crucial role in prevention of infectious diseases and helps in providing long term immunity. Therefore, development of specific vaccines is important in elimination of infectious diseases. The high proportion of chronic infection that is acquired during childhood can be prevented by routine infant immunization program. Considering the safety, cost and availability of inactivated or attenuated whole virus vaccines, focus on finding out the epitopic region within the polyprotein of JEV and vaccine preparation that can promote $B$ cell activity seems to be more effective and easily affordable to the people in low income countries. Conventional vaccines require multiple recall injection at approximately time intervals to attain optimal immune response. Adjuvant is a component that improves the effectiveness of vaccines by inducing robust immune responses. Therefore, adjuvants are used in vaccine formulations as they augment the immune response of any antigen by delivering in native form. Low cost adjuvants should be used to make the vaccine affordable to the people of low income countries. Especially, the conversion of multiple administration vaccine into single administration vaccine may represent an improved advancement towards the betterment of human health care and welfare.

In development of vaccines significant consideration should be given in selecting endemic/local strain for potential vaccine candidates. Systematic planning and evaluation of the experiments, sophisticated infrastructure/facilities (BSL-1 to 4) and well trained professionals can reduce the time and cost of the vaccines. The cost effective vaccine should have global acceptance, accessibility, affordability. The awareness programmers should be conducted in large scale to eradicate the disease and we need to proceed with sense of urgency. 


\section{ACKNOWLEDGMENT}

Researchers are grateful to Director, CCMB and Council of Scientific and Industrial Research (CSIR), India for the encouragement and support for this work. SK Saxena is also supported by NIH Awards (R37DA025576; R01MH085259).

\section{REFERENCES}

Halstead, S.B. and S.J. Thomas, 2011. New Japanese encephalitis vaccines: Alternatives to production in mouse brain. Expert Rev. Vaccines, 10: 355-364. DOI: $10.1586 /$ erv.11.7

Hills, S., R. Martin, A. Marfin and M. Fischer, 2014. Control of Japanese encephalitis in Asia: The time is now. Expert Rev. Anti. Infect. Ther., 12: 901-904. DOI: $10.1586 / 14787210.2014 .929498$

Ishikawa, T., A. Yamanaka and E. Konishi, 2014. A review of successful flavivirus vaccines and the problems with those flaviviruses for which vaccines are not yet available. Vaccine, 32: 1326-1337. DOI: 10.1016/j.vaccine.2014.01.040

Jelinek, T., 2013. IXIARO (®) updated: overview of clinical trials and developments with the inactivated vaccine against Japanese encephalitis. Expert Rev. Vaccines, 12: 859-869. DOI: 10.1586/14760584.2013.835638

Kaur, R. and S. Vrati, 2003. Development of a recombinant vaccine against Japanese encephalitis. J. Neurovirol., 9: 421-431. DOI: $10.1080 / 13550280390218454$

Konishi, E., 2013. Memory B cells: A proposed new immunological correlate for protective efficacy of Japanese encephalitis vaccine. Expert Rev. Vaccines, 12: 871-873. DOI: 10.1586/14760584.2013.814828

McArthur, M.A. and M.R. Holbrook, 2011. Japanese encephalitis vaccines. J. Bioterror. Biodef., 25: S1: 2. DOI: $10.4172 / 2157-2526 . S 1-002$

Monath, T.P., 2002. Japanese encephalitis vaccines: Current vaccines and future prospects. Curr Top Microbiol Immunol., 267: 105-138. PMID: 12082985
Okada, K., T. Iwasa, J. Namazue, M. Akechi and S. Ueda, 2012. JE vaccine clinical study group. Safety and immunogenicity of a freeze-dried, cell culturederived Japanese encephalitis vaccine (Inactivated) (JEBIK $\square$ V) in children. Vaccine, 30: 5967-5972. DOI: $10.1016 /$ j.vaccine.2012.07.034

Plesner, A.M., 2003. Allergic reactions to Japanese encephalitis vaccine. Immunol Allergy Clin. North Am., 23: 665-697. Review. Erratum in: Immunol Allergy Clin North Am. 24: 335. PMID: 14753386.

Verma, R., 2012. Japanese encephalitis vaccine: Need of the hour in endemic states of India. Hum. Vaccin. Immunother, 8: 491-493. DOI: 10.4161/hv.18925

Wilder-Smith, A. and S.B. Halstead, 2010. Japanese encephalitis: update on vaccines and vaccine recommendations. Curr. Opin. Infect. Dis., 230: 426-431. DOI: 10.1097/QCO.0b013e32833c1d01

Yasri, S. and V. Wiwanitkit, 2014. Finding of B cell epitope within polyprotein of Japanese encephalitis virus: A clue for development of new Japanese encephalitis vaccine. Arq Neuropsiquiatr., 72: 478. PMID: 24964123

Yun, S.I. and Y.M. Lee, 2014. Japanese encephalitis: The virus and vaccines. Hum. Vaccin. Immunother, 10: 263-279. DOI: $10.4161 /$ hv.26902

Yun, S.I., B.H. Song, J.K. Kim, G.N. Yun and E.Y. Lee et al., 2014. A molecularly cloned, live-attenuated Japanese Encephalitis Vaccine SA14-14-2 virus: A conserved single amino acid in the ij hairpin of the viral E glycoprotein determines neurovirulence in mice. PLoS Pathog., 10: e1004290. DOI: 10.1371/journal.ppat. 1004290

Halstead, S.B. and S.J. Thomas, 2010. Japanese encephalitis: New options for active immunization. Clin. Infect. Dis., 50: 1155-1164. DOI: $10.1086 / 651271$ 